IRRITABLE BOWEL SYNDROME

\title{
Acute tryptophan depletion affects brain-gut responses in irritable bowel syndrome patients and controls
}

\author{
T O C Kilkens, A Honig, M A van Nieuwenhoven, W J Riedel, R-J M Brummer
}

Gut 2004;53:1794-1800. doi: 10.1136/gut.2004.041657

See end of article for authors' affiliations

\section{Correspondence to:} Ms T O C Kilkens, Maastricht University, Department of Psychiatry and Neuropsychology, Dr Tanslaan 10, $6229 \mathrm{ET}$, Maastricht, the

Netherlands; T.Kilkens@

pn.unimaas.nl

Revised version received 15 April 2004

Accepted for publication

11 May 2004
Background: Serotonin, a key denominator of the brain-gut axis, is involved in the regulation of gastrointestinal motility, secretion, and perception as well as cognition and mood.

Aim: To assess the effects of an acutely lowered serotonin synthesis, using the acute tryptophan depletion (ATD) method, on visceral perception, affective memory performance, and mood in diarrhoea predominant irritable bowel syndrome patients (d-IBS) and controls.

Methods: In a randomised, double blind, crossover design, 14 d-IBS patients and fourteen matched controls were studied under ATD and placebo conditions, respectively. Perception of urge and pain was scored during rectal distensions. Affective memory performance, mood, and biochemical parameters of serotonergic metabolism were simultaneously assessed.

Results: ATD significantly decreased plasma tryptophan (67.0 (2.0) $\vee 24.9(2.0) \mu \mathrm{mol} / \mathrm{l})$ and 5-hydroxyindole acetic acid concentrations (29.9 (1.0) v $15.8(0.6) \mathrm{nmol} / \mathrm{l})$. ATD was associated with significantly increased urge scores specifically in the lower pressure range and overall increased pain scores. ATD significantly lowered the perceptual threshold for first perception compared with placebo (patients $10.6(1.2) \vee 13.6(0.8) \mathrm{mm} \mathrm{Hg}$, controls $12.6(1.3) \vee 15.7(1.2) \mathrm{mm} \mathrm{Hg}$ ) but not for maximal tolerable discomfort (patients 50.5 (3.6) v $51.6(3.3) \mathrm{mm} \mathrm{Hg}$, controls 50.9 (3.3) v $48.8(2.9) \mathrm{mm} \mathrm{Hg}$ ). ATD induced a significant shift in affective memory bias towards preferential loss of positive material but no significant changes in mood. ATD did not differentially affect the patient or control group. Conclusions: We have provided evidence that serotonergic modulation by ATD affects both visceral perception as well as cognition in $\mathrm{d}-\mathrm{IBS}$ and controls. Simultaneous measurement of brain and gut function and the application of ATD contribute to the elucidation of the complex pathophysiology of IBS.
$\mathrm{T}$ he pathophysiology of irritable bowel syndrome (IBS) is not fully understood. A multicomponent conceptual model of IBS has been postulated, involving physiological, affective, cognitive, and behavioural factors. ${ }^{1}$ The "braingut axis" is a theoretical model describing the bidirectional neural pathways linking cognitive and emotional centres in the brain to neuroendocrine centres, the enteric nervous system, and the immune system, and plays a major role in the concept of IBS. ${ }^{2}$ IBS is associated with visceral hypersensitivity and with a high co-occurrence of psychiatric symptoms, in particular affective dysregulation..$^{3-5}$

Serotonin (5-hydroxytryptamine (5-HT)) is a biogenic amine that functions as a neurotransmitter and is located predominantly in the gastrointestinal tract $(80 \%$ of body 5 -HT). Approximately $5 \%$ is located in the brain and cerebrospinal fluid (CSF). 5-HT is peripherally involved in the regulation of gastrointestinal secretion, motility, and perception whereas in the central nervous system (CNS) it plays a role in the regulation of mood and cognition. ${ }^{67}$ Manipulation of serotonergic activity by application of 5-HT modulators has been used in the treatment of both affective disorders and IBS. ${ }^{8-11}$ Hence 5-HT is a key denominator of the "brain-gut axis". ${ }^{10} 12$

Disturbed serotonergic metabolism seems especially prevalent in the diarrhoea predominant type of IBS (d-IBS). ${ }^{13-15}$ Increased postprandial plasma 5-HT levels in d-IBS patients and increased numbers of 5-HT containing enterochromaffin (EC) cells in postinfectious IBS (PI-IBS) have been observed. ${ }^{5} 1617$ In contrast with the known effects of 5-HT on gastrointestinal motility and secretion, the role of 5-HT in visceral perception to volume based distensions has been less well established. ${ }^{718}$
An acute decrease in 5-HT synthesis can be achieved by means of the acute tryptophan depletion (ATD) method. ATD selectively lowers 5-HT synthesis, between four and seven hours after oral administration of an amino acid mixture devoid of the 5-HT precursor tryptophan. ${ }^{19}$ In human subjects, substantial reductions in brain 5-HT synthesis and decreased levels of CSF 5-hydroxyindole acetic acid (5-HIAA), the major metabolite of 5-HT, have been demonstrated. ${ }^{20}$ Peripherally, the ATD method leads to a $60-70 \%$ decrease in plasma tryptophan levels. ${ }^{21}$

ATD has been used over the past decade in the psychiatric setting to investigate the role of the central-5-HT system in patients with affective disorders (depression and anxiety) and their first degree relatives. It serves as a biological model for inducing symptoms of depression such as impaired mood and affective memory performance (loss of words with a positive connotation). ${ }^{22-24}$ In addition, recent findings at our department showed that ATD also affects gastrointestinal physiology by delaying gastric emptying in healthy females. ${ }^{25}$

The above findings indicate that the ATD method offers a potential technique to study the contribution of 5-HT to regulation of the "brain-gut axis". This paper describes the effects of serotonergic modulation, using the ATD method, at

Abbreviations: ATD, acute tryptophan depletion; CNS, central nervous system; CSF, cerebrospinal fluid; IBS, irritable bowel syndrome; d-IBS, diarrhoea predominant irritable bowel syndrome; PI-IBS, postinfectious irritable bowel syndrome; EC, enterochromaffin; 5-HIAA, 5-hydroxyindole acetic acid; LNAAs, large neutral amino acids; 5-HT, 5-hydroxytryptamine; ppp, platelet poor plasma; POMS, profile of mood states; $\mathrm{MINI}$, mini international neuropsychiatric interview; HAM, Hamilton depression rating scale; SCL, symptom checklist; HADS, hospital anxiety and depression rating scale 
Table 1 Subject characteristics (mean (SEM))

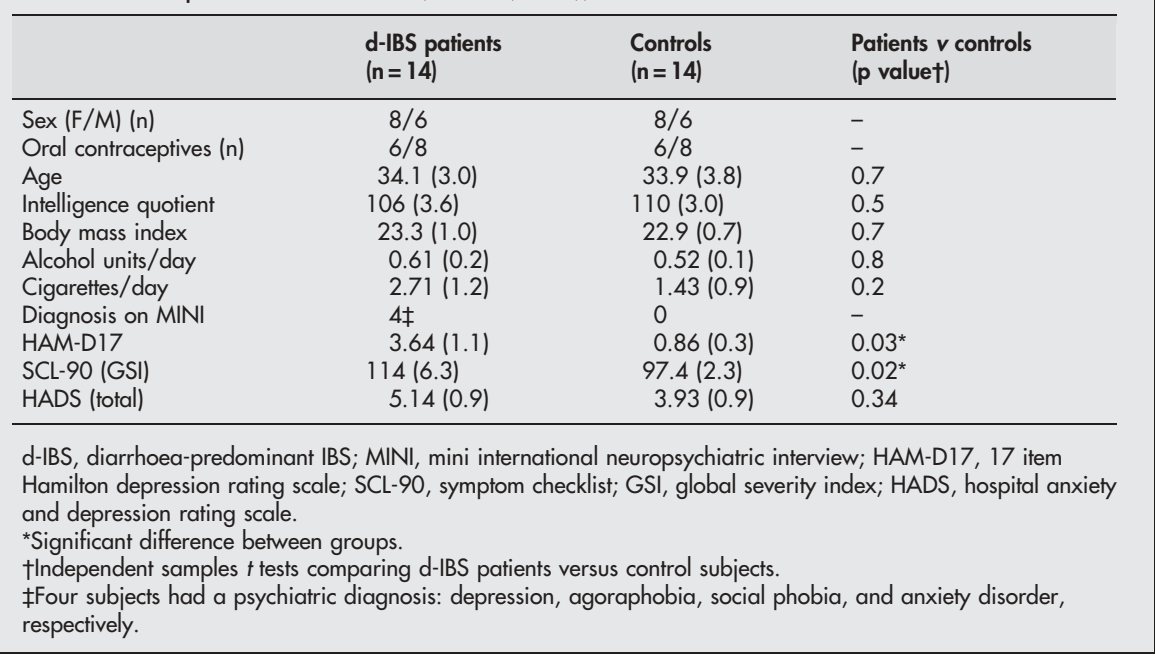

the level of both the brain and gut, in patients with d-IBS and healthy controls. We applied this novel model to investigate whether: (1) ATD influences visceral perception during rectal distensions and (2) whether ATD simultaneously influences affective memory performance and mood. Based on the known effects of ATD on the CNS and given the lines of evidence that (1) IBS is associated with enhanced visceral perception ${ }^{4}$ and lowered mood ${ }^{10}$ and (2) lowered mood is associated with decreased serotonergic activity, ${ }^{6}$ we hypothesised that decreased serotonergic synthesis would be associated with enhanced visceral perception and impaired affective memory performance and mood.

\section{MATERIALS AND METHODS}

\section{Subjects (table 1)}

All subjects were screened which involved a standardised psychiatric examination using the mini international neuropsychiatric interview (MINI) ${ }^{26}$ to determine the present psychiatric state. General psychological state was assessed using the 17 item Hamilton depression rating scale (HAMD17), ${ }^{27}$ the Dutch version of the symptom checklist (SCL90), ${ }^{28}$ and the hospital anxiety and depression rating scale (HADS). ${ }^{29}$ Physical health was assessed by means of a standard physical examination and a urine test. A pregnancy test was performed in all female subjects. Intelligence was estimated using the Groninger intelligence test. ${ }^{30}$ Prior to the test days a training version of the affective memory performance test was practiced to minimise learning effects.

The medical ethics committee of the University Hospital Maastricht approved the study protocol and all subjects gave written informed consent before the start of the study. The required number of included subjects was based on pilot experiments and an estimated effect size of 1.0 (calculated from the standardised mean difference between ATD and placebo conditions with respect to the primary outcome

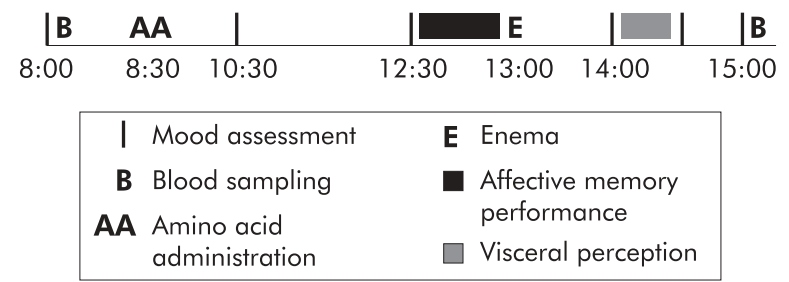

Figure 1 Schematic representation of a test day (flow chart). parameter), with $\beta=0.2$ and $\alpha=0.05$. Fourteen patients fulfilled the diagnosis of d-IBS according to the Rome II criteria, diagnosed by an experienced gastroenterologist. ${ }^{31}$ Exclusion criteria were abdominal surgery (other than appendicectomy and cholecystectomy), use of medications other than oral contraceptives within 14 days prior to testing, lactose intolerance, ${ }^{32}$ a positive first degree psychiatric family history, ${ }^{21}$ any history of psychiatric disease or use of psychoactive medication, premenstrual syndrome, dieting, pregnancy, or lactation, excessive alcohol intake $\quad>20$ alcoholic consumptions a week), and hypertension (diastolic $>100 \mathrm{~mm} \mathrm{Hg}$, systolic $>170 \mathrm{~mm} \mathrm{Hg}$ ), respectively.

Fourteen healthy matched control subjects were included. The same exclusion criteria were used. Additional exclusion criteria for the control subjects were current or history of gastrointestinal disorder, current psychiatric or psychological symptomatology defined as a diagnosis on the MINI, HAMD17 score above 18, global severity index score on the SCL-90 for females $\geqslant 150$ and for males $\geqslant 131$, or HADS scores $\geqslant 8$.

\section{Study design (fig 1)}

The study was conducted following a randomised, placebo controlled, double blind, crossover design. The two test days were separated by a minimum of seven days. All women were tested in the follicular phase of the menstrual cycle or while taking oral contraception. ${ }^{33}$ All subjects were tested within three months to avoid possible seasonal variation. ${ }^{34}$ They were asked to abstain from heavy physical exercise and consumption of alcoholic beverages the day prior to their visit. All subjects attended the laboratory twice after an overnight fast (after 10:00 pm no eating, drinking, or smoking was allowed) at 8:00 am. At $\mathrm{t}=8: 30$ the amino acid drink (ATD or placebo mixture) was administered. Blood samples were taken at $\mathrm{t}=8: 00$ and $\mathrm{t}=15: 00$. Affective memory performance and visceral perception were assessed at $\mathrm{t}=12: 30$ and $\mathrm{t}=14: 00$, respectively. Mood was assessed six times during each test day at $t=8: 00,10: 30,12: 30,14: 00$, $14: 30$, and $t=15: 00$.

\section{Tryptophan depletion}

The Department of Pharmacy of the University Hospital prepared the amino acid mixtures. The tryptophan deficient amino acid (ATD) mixture consisted of 15 amino acids, including five large neutral amino acids (LNAAs). ${ }^{21}$ The placebo mixture contained the same amino acids plus $3 \mathrm{~g}$ of tryptophan in order to prevent a decrease in tryptophan levels. ${ }^{21}$ The mixture was dissolved in $200 \mathrm{ml}$ of tap water and 
subjects were instructed to ingest the mixture as quickly as possible.

\section{Visceral perception}

Bowel preparation consisted of a tap water enema administered one hour before the barostat procedure. The rectal probe was lubricated and placed into the rectum so that the attached end of the bag was $3 \mathrm{~cm}$ from the anal sphincter. The probe consisted of a $700 \mathrm{ml}$ polyethylene bag secured on a rectal catheter (external diameter $=18$ French). The catheter was connected to the electronic distension device (Electronic Barostat, Distender Series II; G\&J Electronics Inc., Toronto, Ontario, Canada) using the Protocol Plus Deluxe Software (G\&J Electronics Inc.). The barostat inflation rate was $50 \mathrm{ml} / \mathrm{s}$ to a constant pressure plateau. Subjects were not given any auditory or visual clues that they were to receive a stimulus and were not told the exact nature of the distension protocol. To reduce the influence of adipose tissue mass and abdominal wall tone, subjects were all placed in the left lateral decubitus position.

In order to unfold the rectal probe, a five minute $10 \mathrm{~mm} \mathrm{Hg}$ distension followed by a one minute $20 \mathrm{~mm} \mathrm{Hg}$ distension was administered. Subsequently, the barostat protocol consisted of intermittent semi-random staircase distensions of 60 seconds duration $(15,10,25,20 \mathrm{~mm} \mathrm{Hg}$, etc) separated by an interval of 30 seconds of baseline pressure. ${ }^{35}$ The end point to stop the series of distensions was the perceptual threshold for maximal tolerable discomfort or if the safety value of the maximal volume of $600 \mathrm{ml}$ was exceeded. During each distension (after 13 seconds of distension) subjects were asked to report their perception of urge and pain. Urge was scored on a panel (Deluxe Perception Panel; G\&J Electronics Inc.) with six buttons labelled: 1, not perceptible; 2 , first perception; 3, minor urge to defecate; 4, normal urge to defecate; 5 , strong urge to defecate; and 6, maximal tolerable urge. ${ }^{35}$ Pain was scored using a $100 \mathrm{~mm}$ visual analogue scale (no pain-maximal tolerable pain). Rectal volumes were measured at the end of each distension (after 60 seconds of distension). Volume was corrected for air compressibility. Rectal compliance $(\Delta \mathrm{V} / \Delta \mathrm{P}$; $\mathrm{P}=10-45 \mathrm{~mm} \mathrm{Hg}$ ) was estimated for each subject.

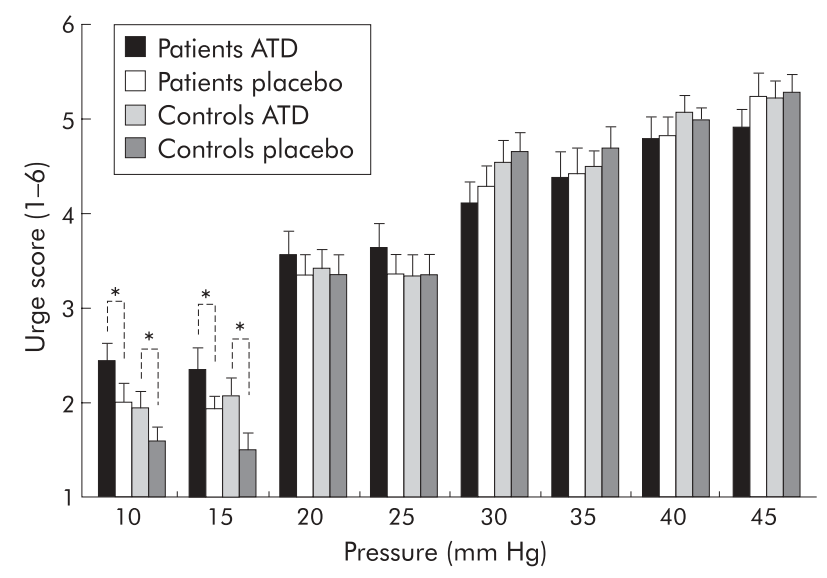

Figure 2 Pressure-urge scores (mean (SEM)) during intermittent pressure distension of the rectum in diarrhoea predominant irritable bowel syndrome (d-IBS) patients and control subjects, during acute tryptophan depletion (ATD) and placebo. Overall, ATD did not significantly affect urge scores compared with placebo. However, post hoc analysis indicated that in the lower pressure range, ATD was significantly associated with increased urge scores compared with placebo $(p<0.0001)(10-15 \mathrm{~mm} \mathrm{Hg})$.

\section{Cognition and mood}

The affective memory test consisted of a list of 30 emotionally loaded stimulus words ( 12 positive, 12 negative, and six neutral). ${ }^{23}$ All 30 words on the list were consecutively presented three times on a computer screen. Each presentation of the list ended with a free recall of the words. Recall scores were summed to comprise the total immediate recall score. After the third presentation, subjects performed a 30 minute distraction task and subsequently subjects were requested to recall as many of the previously learned words as possible (delayed recall). Outcome measures were the percentages of positive, negative, and neutral words recalled of the total immediate recall and delayed recall score..$^{22}$ Mood was assessed with visual analogue scales adapted from the profile of mood states (POMS). ${ }^{36}$ It consisted of 32 items describing bipolar mood adjectives (for example, happy-sad). Items were grouped to form measures of five mood dimensions: depression, tension, vigour, anger, and fatigue, respectively.

\section{Biochemical parameters}

Total plasma tryptophan concentrations and the tryptophan/ ¿LNAAs ratios (sum of tyrosine, valine, leucine, isoleucine, and phenylalanine concentrations) were determined. This ratio provides an estimate of tryptophan uptake into the brain and consequently central 5 -HT synthesis. ${ }^{37}$ In addition, concentrations of platelet 5-HT, platelet poor plasma (ppp) 5-HT, its major metabolite 5-HIAA, and 5-HT turnover (5-HIAA:5-HT) were determined as markers of peripheral 5-HT metabolism. Blood was sampled in EDTA Vacutainer tubes. Samples for tryptophan and LNAAs were immediately placed on ice and centrifuged within 30 minutes ( 10 minutes, $900 \mathrm{~g}, 4^{\circ} \mathrm{C}$ ). For platelet 5 -HT measurement, whole blood was used. In addition, an aliquot of blood kept at room temperature was used for whole blood platelet counting. Samples for ppp 5-HT and 5-HIAA were kept at room temperature and centrifuged $\left(20\right.$ minutes, $\left.2600 \mathrm{~g}, 20^{\circ} \mathrm{C}\right)$. As a control of the ppp preparation procedure, platelets were also counted in the ppp using a Coulter Counter (Coulter MD Series, Coulter Corporation, Hialeah, Florida, USA). All samples were stored at $-80^{\circ} \mathrm{C}$ until analysis. Plasma tryptophan, LNAAs, ${ }^{38} 39$ ppp 5-HT, and 5-HIAA ${ }^{40}{ }^{41}$ samples were analysed using high performance liquid chromatography, as previously described.

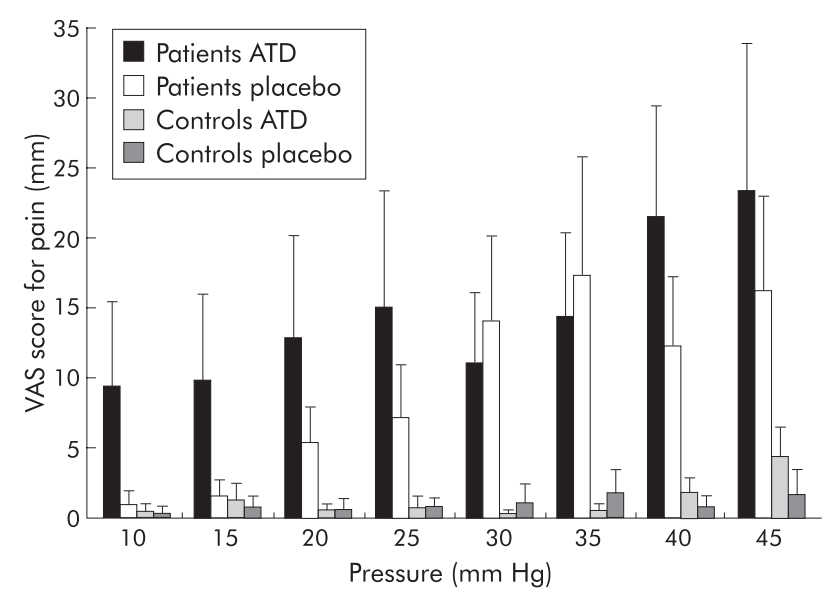

Figure 3 Pressure-pain scores (mean (SEM)) during intermittent pressure distension of the rectum in diarrhoea predominant irritable bowel syndrome (d-IBS) patients and control subjects, during acute tryptophan depletion (ATD) and placebo. Overall, patients showed significantly increased pain scores compared with controls $(p=0.02)$. ATD was significantly associated with increased pain scores $(p=0.04)$. 
Table 2 Perceptual thresholds (mean (SEM)) in diarrhoea predominant irritable bowel syndrome (d-IBS) patients and control subjects during acute tryptophan depletion (ATD) and placebo

\begin{tabular}{lllll}
\hline & & d-IBS patients & Controls & $\begin{array}{l}\text { ATD v placebo } \\
\text { (p value) }\end{array}$ \\
\hline $\begin{array}{l}\text { Pressure threshold }(\mathrm{mm} \mathrm{Hg}) \\
\text { First perception }\end{array}$ & ATD & $10.6(1.2)$ & $12.6(1.3)$ & $0.003^{*}$ \\
Maximal tolerable discomfort & Placebo & $13.6(0.8)$ & $15.7(1.2)$ & 0.8 \\
& ATD & $50.5(3.6)$ & $50.9(3.3)$ & 0.8 \\
& Placebo & $51.6(3.3)$ & $48.8(2.9)$ & \\
\hline
\end{tabular}

\section{Statistical analysis}

The primary outcome parameter was visceral perception. Secondary outcome parameters were rectal compliance, affective memory performance, and mood. Data are presented as mean (SEM). Differences in subject characteristics were analysed using independent samples $t$ tests. Outcome variables were analysed using analysis of variance design (ANOVA) for repeated measures. For visceral perception thresholds (first perception and maximal tolerable discomfort) and affective memory performance, the within subjects factor was treatment (two levels: ATD, placebo). For biochemical parameters, the within subjects factors were treatment (two levels: ATD, placebo) and time (two levels: $t=8: 00,15: 00)$. Urge and pain perception were analysed in the range $10-45 \mathrm{~mm} \mathrm{Hg}$, as our total number of subjects became too small for adequate statistical analysis at pressures above $45 \mathrm{~mm} \mathrm{Hg}$. For visceral urge, pain perception, and mood analyses, within subjects factors were treatment (two levels: ATD, placebo), pressure (eight levels: 10, 15, 20, 25, 30, 35, 40, $45 \mathrm{~mm} \mathrm{Hg}$ ) and time (six levels: $\mathrm{t}=8: 00$, 10:30, $12: 30,14: 00,14: 30$, and 15:00). The between subjects factor for all outcome variables was diagnosis of d-IBS (two levels: d-IBS, controls). In case of a slight violation of sphericity, the Greenhouse-Geisser correction was applied. If indicated, post hoc analyses were performed for certain time points and Bonferroni corrections were applied. A p value of less than 0.05 was considered statistically significant. Statistical analyses were performed using the SPSS 10.0 for Windows software package.

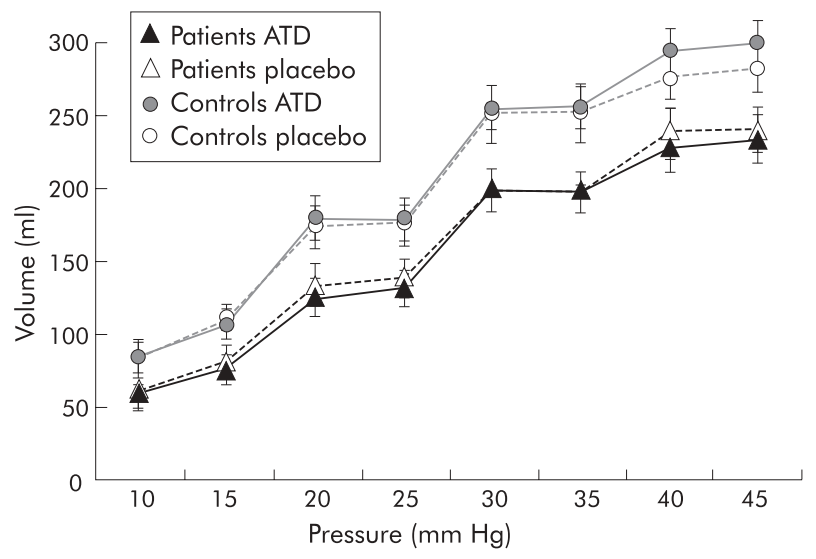

Figure 4 Pressure-volume curves (mean (SEM)) during intermittent pressure distension of the rectum in diarrhoea predominant irritable bowel syndrome (d-IBS) patients and control subjects, during acute tryptophan depletion (ATD) and placebo. Patients showed significantly decreased rectal volumes compared with controls $(p=0.02)$. ATD did not significantly influence pressure-volume relations.

\section{RESULTS}

\section{Visceral perception}

Urge and pain (figs 2, 3)

Urge and pain scores increased significantly with increasing pressure $(\mathrm{F}(7,13)=199.0, \mathrm{p}<0.0001 ; \mathrm{F}(7,12)=7.1, \mathrm{p}<0.01$, respectively). For urge scores, there was a strong significant pressure $\times$ diagnosis of d-IBS interaction $(F(7,133)=3.2$, $\mathrm{p}<0.005$ ), indicating that patients had relatively higher urge scores in the lower pressure range and relatively lower urge scores in the higher pressure range compared with controls (fig 2). Post hoc analysis indicated that in the lower pressure range (10-15 $\mathrm{mm} \mathrm{Hg}$ ), patients had significantly higher urge scores compared with controls $(\mathrm{F}(1,26)=4.6, \mathrm{p}<0.05)$.

Patients experienced overall significantly more pain compared with controls $(\mathrm{F}(1,17)=4.7, \mathrm{p}<0.05)$ and their pain scores increased significantly more with increasing pressure compared with controls (pressure $\times$ diagnosis of d-IBS interaction $)(F(9,119)=7.1, p<0.0001)$, which confirms the presence of hypersensitivity in our d-IBS population.

Overall, there was a significant pressure $\times$ treatment interaction for the perception of urge $(F(7,133)=3.1, p<0.01)$, indicating that in the lower pressure range ATD enhanced the perception of urge whereas this enhancing effect of ATD was no longer present in the higher pressure range (fig 2). Post hoc analysis showed significantly increased urge scores in the lower pressure range (10-15 mm $\mathrm{Hg}$ ) during ATD compared with placebo $(\mathrm{F}(1,26)=23.6, \mathrm{p}<0.0001)$.

ATD was significantly associated with increased overall pain scores compared with placebo $(\mathrm{F}(1,17)=3.4, \mathrm{p}<0.05)$. For the pain scores there was, in contrast with the urge scores, no significant pressure $\times$ treatment interaction $(\mathrm{p}>0.3)$, indicating that the effect of ATD was not specific for the higher or lower pressure range (fig 3). The effects of ATD compared with placebo on urge and pain scores did not differ significantly between patients and controls.

\section{Perceptual thresholds (table 2)}

Patients tended to have lower first perception thresholds compared with controls although this did not reach significance $(\mathrm{F}(1,26)=2.5, \mathrm{p}=0.06)$. Thresholds for maximal tolerable discomfort did not differ between patients and controls $(\mathrm{F}(\mathrm{l}, 26)=0.1, \mathrm{p}=0.8)$.

ATD significantly lowered the perceptual threshold for first perception compared with placebo $(\mathrm{F}(1,26)=9.03$, $\mathrm{p}=0.003)$. ATD did not significantly influence the perceptual threshold for maximal tolerable discomfort $(F(1,26)=0.06$, $\mathrm{p}=0.8$ ) (table 2). The effects of ATD compared with placebo on perceptual thresholds did not differ significantly between patients and controls $(\mathrm{p}>0.4)$.

\section{Rectal compliance (fig 4)}

The pressure-volume curves differed significantly between patients and controls $(\mathrm{F}(\mathrm{l}, 20)=5.7, \mathrm{p}<0.05)$ (fig 4). There was no significant difference in pressure-volume relation or apparent rectal compliance during ATD compared with 
Table 3 Influence of acute tryptophan depletion (ATD) on affective memory performance in diarrhoea predominant irritable bowel syndrome (d-IBS) patients and control subjects

\begin{tabular}{|c|c|c|c|c|}
\hline & & d-IBS patients & Controls & $\begin{array}{l}\text { ATD } v \text { placebo } \\
\text { ( } p \text { value) }\end{array}$ \\
\hline \multicolumn{5}{|c|}{ Total immediate recall } \\
\hline \multirow[t]{2}{*}{ Positive words } & ATD & $38.4(4.1)$ & $42.4(4.2)$ & 0.06 \\
\hline & Placebo & $45.3(3.7)$ & $48.9(4.4)$ & \\
\hline \multirow[t]{2}{*}{ Negative words } & ATD & $41.7(2.2)$ & $44.4(3.3)$ & 0.86 \\
\hline & Placebo & $42.0(4.2)$ & $43.4(4.3)$ & \\
\hline \multirow[t]{2}{*}{ Neutral words } & ATD & $44.8(4.3)$ & $50.1(5.2)$ & 0.20 \\
\hline & Placebo & $46.9(4.4)$ & $56.6(3.8)$ & \\
\hline \multicolumn{5}{|l|}{ Delayed recall } \\
\hline \multirow[t]{2}{*}{ Positive words } & ATD & $31.6(5.6)$ & $43.5(7.4)$ & $<0.005^{*}$ \\
\hline & Placebo & $46.1(5.6)$ & $52.9(7.0)$ & \\
\hline \multirow[t]{2}{*}{ Negative words } & ATD & $41.4(4.7)$ & $45.3(6.1)$ & 0.40 \\
\hline & Placebo & $44.0(6.8)$ & $48.2(7.3)$ & \\
\hline \multirow[t]{2}{*}{ Neutral words } & ATD & $34.6(5.9)$ & $57.5(8.4)$ & 0.31 \\
\hline & Placebo & $48.6(7.4)$ & $57.9(8.7)$ & \\
\hline
\end{tabular}

Mean (SEM) percentages of positive, negative, and neutral words recalled immediately (total immediate recall) and recalled after a delay of 30 minutes (delayed recall).

*Significant after Bonferroni correction.

placebo $(F(1,20)=0.09, p=0.8 ; F(1,26)=0.03, p=0.9$, respectively).

\section{Cognition and mood (table 3)}

Immediate and delayed memory performance as well as percentages of recalled positive and negative words did not significantly differ between patients and controls $(\mathrm{p}>0.3)$. However, patients tended to have greater loss of delayed recalled neutral words compared with controls $(\mathrm{F}(\mathrm{l}$, 26) $=9.5, \mathrm{p}=0.06)($ table 3$)$.

ATD was significantly associated with impaired immediate and delayed recall performance $(\mathrm{F}(1,26)=3.3, \mathrm{p}<0.05$; $\mathrm{F}(1,26)=9.5, \mathrm{p}<0.005$, respectively) compared with placebo. Impaired delayed recall was due to impaired recall of positive words $(F(1,26)=10.7, p<0.005)$. Delayed recall of negative and neutral words was not significantly affected by ATD.

ATD did not differentially affect the patient or control group $(p>0.3)$. There were no significant differences in mood between groups or treatment conditions ( $p$ values $>0.2$ ).

\section{Biochemical parameters (table 4)}

Biochemical parameters did not differ significantly between groups or treatment conditions at baseline. There were no significant correlations between baseline biochemical values and perception scores under placebo conditions. Tryptophan concentrations as well as tryptophan $/ \Sigma$ LNAAs ratios decreased significantly over time during ATD compared with placebo $\quad(F(1,26)=252.5, \quad p<0.0001 ; \quad F(1,26)=164.8$, $\mathrm{p}<0.000 \mathrm{l}$, respectively). ATD did not significantly influence platelet 5-HT or ppp 5-HT concentrations (table 3). 5-HIAA concentrations and ppp 5-HT turnover decreased significantly under ATD conditions compared with placebo $(\mathrm{F}(\mathrm{l}$, $26)=338, \mathrm{p}<0.0001 ; \mathrm{F}(1,26)=11, \mathrm{p}<0.01$, respectively $)$. The change in biochemical parameters induced by ATD did not significantly differ between patients and controls $(p>0.5)$ although a trend towards a differential effect of ATD on ppp 5 -HT concentrations was observed $(\mathrm{F}(1,26)=32, \mathrm{p}=0.09)$.

\section{DISCUSSION}

We have demonstrated for the first time that acute tryptophan depletion (ATD) affects both visceral perception and cognition in d-IBS patients and healthy controls. In addition, we have described an experimental model which can detect changes in the "brain-gut axis" due to acute alteration of serotonergic activity.

The role of specific 5-HT modulators, mainly 5- $\mathrm{HT}_{3}$ antagonists and $5-\mathrm{HT}_{4}$ agonists, in visceral perception has been the subject of earlier studies. ${ }^{72}$ However, it is not feasible to compare our results with studies using receptor specific 5-HT modulators as both at the CNS as well as the gastrointestinal level 5-HT interacts with a number of different 5-HT receptor subclasses that are either stimulatory or inhibitory. It is not known how ATD specifically influences these subreceptors. Data concerning the role of the

Table 4 Biochemical parameters at $t=8: 00$ and $t=15: 00$ (mean (SEM)) during acute tryptophan depletion (ATD) and placebo, in the diarrhoea predominant irritable bowel syndrome (d-IBS) patients and control subjects

\begin{tabular}{|c|c|c|c|c|c|c|}
\hline & & \multicolumn{2}{|c|}{ d-IBS patients } & \multicolumn{2}{|l|}{ Controls } & \multirow{2}{*}{$\begin{array}{l}\text { ATD v placebo } \\
\text { (p value) }\end{array}$} \\
\hline & & $t=8: 00$ & $t=15: 00$ & $t=8: 00$ & $t=15: 00$ & \\
\hline \multirow[t]{2}{*}{ Tryptophan ( $\mu \mathrm{mol} / \mathrm{l})$} & ATD & $65.6(3.0)$ & $23.1(2.4)$ & $68.4(2.7)$ & $26.7(3.1)$ & $<0.0001$ \\
\hline & Placebo & $62.8(2.0)$ & $91.0(6.0)$ & $65.2(2.1)$ & $100(5.7)$ & \\
\hline \multirow[t]{2}{*}{ 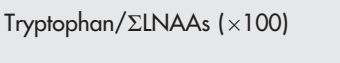 } & ATD & $11.9(2.5)$ & $2.30(1.0)$ & $12.1(2.6)$ & $2.30(0.7)$ & $<0.0001$ \\
\hline & Placebo & $11.4(2.4)$ & $9.70(2.1)$ & $11.4(2.0)$ & $10.3(2.9)$ & \\
\hline \multirow[t]{2}{*}{ Platelet $5-\mathrm{HT}$ (nmol $/ 10^{9}$ platelets) } & ATD & $4.40(0.4)$ & $4.80(0.5)$ & $5.70(0.7)$ & $5.80(0.8)$ & 0.78 \\
\hline & Placebo & $4.30(0.6)$ & $4.50(0.6)$ & $5.20(0.7)$ & $5.40(0.8)$ & \\
\hline \multirow{2}{*}{ ppp 5-HIAA (nmol/l) } & ATD & $28.9(1.6)$ & 15.6 (1.0) & $30.9(1.1)$ & $16.1(0.8)$ & $<0.0001$ \\
\hline & Placebo & $28.9(1.1)$ & $29.4(1.2)$ & $29.8(1.3)$ & $31.6(1.8)$ & \\
\hline \multirow[t]{2}{*}{ ppp 5-HT (nmol/l) } & ATD & $9.80(1.6)$ & $10.7(1.4)$ & $9.20(1.5)$ & 7.90 (1.1) & 0.25 \\
\hline & Placebo & $10.7(1.7)$ & $10.8(1.7)$ & $9.01(1.2)$ & $11.9(1.5)$ & \\
\hline \multirow[t]{2}{*}{ 5-HT furnover } & ATD & $3.80(0.5)$ & $2.34(0.6)$ & $3.98(0.5)$ & $2.68(0.5)$ & $<0.01$ \\
\hline & Placebo & $3.48(0.5)$ & $3.37(0.6)$ & $3.97(0.5)$ & $3.63(0.6)$ & \\
\hline
\end{tabular}

5-HT, 5-hydroxytryptamine (serotonin); ppp, platelet poor plasma; 5-HIAA, 5-hydroyindole acetic acid; $\Sigma$ LNAAs, large neutral amino acids (sum of tyrosine, valine, leucine, isoleucine, and phenylalanine). 
psychotropic selective serotonin reuptake inhibitors, which enhance the overall availability of 5-HT, on visceral perception are conflicting. Reduced oesophageal sensitivity to distension has been described but these drugs seem to lack this effect at the gastric level. ${ }^{18}$

In contrast with most studies evaluating the therapeutic effects of long term serotonergic modulation on gastrointestinal physiology, this study was designed to investigate the effects of acute serotonergic modulation within the brain-gut axis. This is the first time that the effects of lowered 5-HT synthesis using the ATD method on both brain and gut function have been studied. ATD significantly lowered the perceptual threshold for first perception but not for maximal tolerable discomfort. In addition, ATD was associated with increased urge scores specifically in the lower pressure range and overall increased pain scores. The effect of ATD on pain scores was not pressure dependent. As pain is a nociceptive stimulus, our data seem to be in accordance with the results of others who reported that the induced reduction in IBS symptoms by a $5-\mathrm{HT}_{3}$ antagonist was maximal in the absence of visceral nociceptive input. ${ }^{43}$ As a possible mechanism explaining these findings it was hypothesised that the inhibitory effect of the $5-\mathrm{HT}_{3}$ antagonist on central neuronal excitability can be overcome by activation of nociceptive rectal afferents. Apart from this biological reason, the lack of a pressure dependent effect for the perception of pain may also be due to a methodological problem. A minority of our subjects reported perception of pain before reaching their threshold for maximal tolerable discomfort. Hence this resulted in large variations in our pain data.

The observed memory impairing effects of ATD on delayed recall are similar to former studies applying ATD in patients with mood disorders as well as in healthy controls..$^{21}{ }^{23}$ In addition to impaired memory function, "mood congruence" is one of the features of the depressive syndrome (that is, depressed patients are more likely to store or recall information that is congruent with their mood state). ${ }^{45}$ The presence of a depression-like memory bias in IBS is however not fully established. ${ }^{46}{ }^{47}$ Whitehead and Palsson suggested that psychological response bias resulting in increased attention and hypervigilance to gastrointestinal sensations is present in IBS and influences visceral perception. ${ }^{48}$ In our study, ATD induced a depression-like affective memory bias. We do not know however whether ATD induced increased attention towards gastrointestinal sensations as our word list did not include gastrointestinal annotated words. In future research differentiation between words with and without a gastrointestinal annotation should be made. In our opinion, response bias could potentially have enhanced visceral perception during ATD. This hypothesis integrating cognition in the neurophysiology of visceral perception needs further validation and may have consequences in the clinical practice of IBS. During ATD, we did not find a significant decrease in mood status, using the POMS, in IBS patients or healthy controls. In our opinion however affective memory bias may offer a more objective and sensitive biomarker of central serotonergic dysregulation.

It is difficult to define the primary point of action of ATD on visceral perception. Importantly, 5-HT reuptake by the serotonin reuptake transporter is a mechanism that is relevant in both the digestive tract and CNS. ${ }^{49}$ The fact that ATD lowers central 5-HT synthesis and our findings of ATD induced affective memory bias favour a central influence of ATD. ${ }^{20}{ }^{50}$ In addition, animal studies have shown that an acute decrease in serotonergic activity is associated with increased sensory information processing, which is in concordance with enhanced visceral perception during ATD. ${ }^{51}$

Until now, ATD has only been used with regard to changes in CNS functioning. However, our data also strongly suggest that ATD exerts its metabolic action at the level of the gut. Only plasma tryptophan/LNAAs ratios have been routinely assessed as an indication of central serotonergic depletion. We are the first to measure ppp levels of 5-HT and 5-HIAA in addition to plasma tryptophan levels. The significantly decreased plasma 5-HIAA concentrations strongly suggest that 5-HT metabolism in the gut was decreased because the gut is by far the major $(>55 \%)$ contributor to the total 5-HIAA plasma pool. ${ }^{52}$ Although plasma 5-HT turnover significantly decreased during ATD, this was not reflected by a significant decrease in ppp 5-HT. We hypothesise that blood platelets release 5-HT in order to counterbalance lowered ppp 5-HT concentrations during ATD. The finding that platelet 5-HT was not significantly affected by ATD is in line with evidence that blood platelets act as a 5-HT reservoir and therefore are indicative of long term changes in 5-HT metabolism.

Apparently, relationships between systemic, gastrointestinal, and CNS 5-HT metabolism and the influence of acute and chronic serotonergic modulation on each of these systems needs further study. Although it is of interest to know whether pharmacological and metabolic serotonergic modulation primarily acts at the CNS or peripheral level, one has to keep in mind that the brain-gut axis consists of bidirectional neurohumoral pathways and hence modulation at one level may affect various levels of the brain-gut axis. ${ }^{10}$

Increased postprandial ppp 5-HT levels in d-IBS patients and increased numbers of 5-HT containing EC cells in PI-IBS have been observed. This seems paradoxical to our findings of decreased 5-HT levels associated with enhanced visceral perception. Increased postprandial ppp 5-HT levels have been associated with postprandial symptomatology in d-IBS. ${ }^{13} 16$ However, we did not show increased ppp 5-HT levels in d-IBS. This can be explained by analytical and methodological differences as well as the fact that the subjects in our study were fasted of any nutrients during the five hours before the assessment of visceral perception and ppp 5-HT. Our results suggest a different, although not significant, effect of ATD on ppp 5-HT levels between patients and controls. This may support evidence concerning disturbed serotonergic metabolism in the d-IBS subgroup type of IBS. ${ }^{1314} 16$ Dunlop et al showed that increased EC cells as well as depression are equally important independent predictors of developing PI-IBS. ${ }^{5}$ Gastrointestinal motility may be primarily peripherally determined and our study does not solve the contribution of central versus peripheral 5-HT in the role of altered motility and perception in IBS. Recently, two tryptophan hydroxylase (rate limiting enzyme of 5-HT synthesis) isoforms have been identified which differentially modulate peripheral and central 5-HT effects. ${ }^{53}$ This duality of the 5-HT system may open new avenues for specific investigational and therapeutic approaches exclusively affecting central or peripheral 5-HT actions. ${ }^{54}$

The time window of ATD induced metabolic activity at the CNS level may differ from that at the gut level. However, our cognitive and biochemical results indicated that ATD influenced both the CNS as well as the periphery during our experiments. Our results concern the change in brain-gut interaction due to an acute decrease in serotonergic activity. Whether these findings are also valid in prolonged change in serotonergic activity by pharmacological or nutritional means needs to be investigated. We did not find a significant effect of ATD on rectal compliance. Although our barostat protocol was primarily designed to detect changes in visceral perception, this suggests that acute changes in serotonergic activity differentially affect visceral perception and rectal compliance. The ATD model mimics characteristics of IBS in healthy controls. During ATD, healthy controls had urge scores comparable with those of patients in the placebo condition. 
The fact that ATD did not increase pain scores in the control group to levels observed in the patient group suggest that pain and urge perception are different entities.

Our results support further study of the effect of serotonergic modulation on the brain-gut interaction in IBS. We have provided evidence that serotonergic modulation by ATD affects both visceral perception as well as cognition in d-IBS patients and controls. Simultaneous measurement of brain and gut function and application of ATD may contribute to elucidation of the complex pathophysiology of IBS.

\section{Authors' affiliations}

T O C Kilkens, Brain and Behaviour Institute, and Nutrition and Toxicology Research Institute Maastricht, University Hospital Maastricht, Maastricht, the Netherlands

A Honig, Brain and Behaviour Institute, and Department of Psychiatry, University Hospital Maastricht, Maastricht, the Netherlands M A van Nieuwenhoven, R-J M Brummer, Nutrition and Toxicology Research Institute Maastricht, and Department of Gastroenterology, University Hospital Maastricht, Maastricht, the Netherlands W J Riedel, Brain and Behaviour Institute, University Hospital Maastricht, Maastricht, the Netherlands, and Department of Psychiatry, University of Cambridge, Cambridge, UK

\section{REFERENCES}

1 Talley NJ, Spiller R. Irritable bowel syndrome: a little understood organic bowel disease? Lancet 2002;360:555-64.

2 Mayer EA, Gebhart GF. Basic and clinical aspects of visceral hyperalgesia. Gastroenterology 1994;107:271-93.

3 Walker EA, Roy-Byrne PP, Katon WJ, et al. Psychiatric illness and irritable bowel syndrome: a comparison with inflammatory bowel disease. Am J Psychiatry 1990;147:1656-61.

4 Delvaux M. Role of visceral sensitivity in the pathophysiology of irritable bowel syndrome. Gut 2002;51(suppl 1):i67-71.

5 Dunlop SP, Jenkins D, Neal KR, et al. Relative importance of enterochromaffin cell hyperplasia, anxiety, and depression in postinfectious IBS. Gastroenterology 2003;125:1651-9.

6 Charney DS. Monoamine dysfunction and the pathophysiology and treatment of depression. J Clin Psychiatry 1998;59(suppl 14):11-14.

7 Camilleri M. Serotonergic modulation of visceral sensation: lower gut. Gut 2002:51(suppl 1):i81-6.

8 Creed F, Fernandes L, Guthrie E, et al. The cost-effectiveness of psychotherapy and paroxetine for severe irritable bowel syndrome. Gastroenterology 2003; 124:303-17.

9 Jackson JL, O'Malley PG, Tomkins G, et al. Treatment of functional gastrointestinal disorders with antidepressant medications: a meta-analysis. Am J Med 2000; 108:65-72.

10 Kilkens TO, Honig A, Rozendaal N, et al. Serotonergic modulators in the treatment of irritable bowel syndrome-influence on psychiatric and gastrointestinal symptoms. Aliment Pharmacol Ther 2003;17:43-51.

11 Clouse RE. Antidepressants for irritable bowel syndrome. Gut 2003;52:598-9.

12 Kim DY, Camilleri M. Serotonin: a mediator of the brain-gut connection. Am J Gastroenterol 2000;95:2698-709.

13 Houghton LA, Atkinson W, Whitaker RP, et al. Increased platelet depleted plasma 5-hydroxytryptamine concentration following meal ingestion in symptomatic female subjects with diarrhoea predominant irritable bowel syndrome. Gut 2003;52:663-70.

14 Singh RK, Pandey HP, Singh RH. Correlation of serotonin and monoamine oxidase levels with anxiety level in diarrhea-predominant irritable bowel syndrome. Indian J Gastroenterol 2003;22:88-90.

15 Camilleri M, Northcutt AR, Kong S, et al. Efficacy and safety of alosetron in women with irritable bowel syndrome: a randomised, placebo-controlled trial. Lancet 2000;355:1035-40.

16 Bearcroft CP, Perrett D, Farthing MJ. Postprandial plasma 5 hydroxytryptamine in diarrhoea predominant irritable bowel syndrome: a pilot study. Gut 1998;42:42-6

17 Sanger GJ, Yoshida M, Yahyah M, et al. Increased defecation during stress or after 5-hydroxytryptophan: selective inhibition by the 5-HT(4) receptor antagonist, SB-207266. Br J Pharmacol 2000;130:706-12.

18 Tack J, Sarnelli G. Serotonergic modulation of visceral sensation: upper gastrointestinal tract. Gut 2002;51(suppl 1):i77-80.

19 Moore P, Landolt H-P, Seifritz E, et al. Clinical and physiological consequences of rapid tryptophan depletion. Neuropsychopharmacology 2000;23:601-22

20 Carpenter LL, Anderson GM, Pelton GH, et al. Tryptophan depletion during continuous CSF sampling in healthy human subjects. Neuropsychopharmacology 1998; 19:26-35.

21 Sobczak S, Riedel WJ, Booij I, et al. Cognition following acute tryptophan depletion: difference between first-degree relatives of bipolar disorder patients and matched healthy control volunteers. Psychol Med 2002;32:503-15.
22 Klaassen T, Riedel WJ, Deutz NE, et al. Mood congruent memory bias induced by tryptophan depletion. Psychol Med 2002;32:167-72.

23 Riedel WJ, Klaassen T, Deutz NE, et al. Tryptophan depletion in normal volunteers produces selective impairment in memory consolidation. Psychopharmacology (Berl) 1999;141:362-9.

24 Yatham LN, Liddle PF, Shiah IS, et al. Effects of rapid tryptophan depletion on brain 5-HT(2) receptors: a PET study. Br J Psychiatry 2001;178:448-53.

25 Van Nieuwenhoven MA, Valks SDM, Sobczak S, et al. Acute tryptophan depletion slows gastric emptying in females. Br J Nutr 2004;91:1-6.

26 Sheehan D. MINI international neuropsychiatric interview. Tampa: University of South Florida, 1994.

27 Hamilton M. Development of a rating scale for primary depressive illness. Br J Soc Clin Psychol 1967;6:278-96.

28 Arrindell WA, Ettema JHM. SCL-90. Een multidimensionale psychopathologie indicator [SCL-90. A multidimensional indicator of psychopathology]. Lisse, the Netherlands: Swets and Zeitlinger, 1986

29 Mykletun A, Stordal E, Dahl AA. Hospital anxiety and depression (HAD) scale: factor structure, item analyses and internal consistency in a large population. Br J Psychiatry 2001;179:540-4.

30 Luteijn F, van der Ploeg FAE. Handleiding Groninger Intelligentie test (GIT) [Manual Groninger Intelligence Test]. Lisse, the Netherlands: Swets and Zeitlinger, 1983.

31 Drossman DA, Heaton K, Irvine EJ, et al. Functional bowel disorders and functional abdominal pain. In: Drossman DA, Corazziari E, Talley N, et al. The functional gastrointestinal disorders, Diagnosis, pathophysiology and treatment:A multinational consensus. McLean, VA: Degnon Associates, 2000:351-98.

32 Ledochowski M, Sperner-Unterweger B, Fuchs D. Lactose malabsorption is associated with early signs of mental depression in females: a preliminary report. Dig Dis Sci 1998;43:2513-17.

33 Houghton LA, Lea R, Jackson N, et al. The menstrual cycle affects rectal sensitivity in patients with irritable bowel syndrome but not healthy volunteers. Gut 2002:50:471-4.

34 Maes M, Scharpe S, Verkerk R, et al. Seasonal variation in plasma Ltryptophan availability in healthy volunteers. Relationships to violent suicide occurrence. Arch Gen Psychiatry 1995;52:937-46.

35 Sloots CE, Felt-Bersma RJ, Cuesta MA, et al. Rectal visceral sensitivity in healthy volunteers: influences of gender, age and methods. Neurogastroenterol Motil 2000;12:361-8

36 Mcnair DM, Lorr M, Droppleman LF. Manual for the profile of mood states (POMS) manual. San Diego: Educational and Industrial Testing Service, 1992

37 Bell C, Abrams J, Nutt D. Tryptophan depletion and its implications for psychiatry. Br J Psychiatry 2001;178:399-405.

38 Maes M, Wauters A, Verkerk R, et al. Lower serum L-tryptophan availability in depression as a marker of a more generalized disorder in protein metabolism. Neuropsychopharmacology 1996;15:243-51

39 Turnell DC, Cooper JD. Rapid assay for amino acids in serum or urine by precolumn derivatization and reversed-phase liquid chromatography. Clin Chem 1982:28:527-31.

40 Stronks DL, Tulen JH, Verheij R, et al. Serotonergic, catecholaminergic, and cardiovascular reactions to mental stress in female migraine patients. A controlled study. Headache 1998;38:270-80.

41 Fekkes D, Timmerman L, Pepplinkhuizen L. Effects of clomipramine on plasma amino acids and serotonergic parameters in panic disorder and depression. Eur Neuropsychopharmacol 1997;7:235-9

42 Talley NJ. Review article: 5-hydroxytryptamine agonists and antagonists in the modulation of gastrointestinal motility and sensation: clinical implications. Aliment Pharmacol Ther 1992;6:273-89.

43 Berman SM, Chang L, Suyenobu B, et al. Condition-specific deactivation of brain regions by $5-\mathrm{HT} 3$ receptor antagonist Alosetron. Gastroenterology 2002; 123:969-77.

44 Park SB, Coull JT, McShane RH, et al. Tryptophan depletion in normal volunteers produces selective impairments in learning and memory Neuropharmacology 1994;33:575-88.

45 Elliott R, Rubinsztein JS, Sahakian BJ, et al. The neural basis of moodcongruent processing biases in depression. Arch Gen Psychiatry 2002; 59:597-604

46 Toner BB, Garfinkel PE, Jeejeebhoy KN, et al. Self-schema in irritable bowel syndrome and depression. Psychosom Med 1990;52:149-55.

47 Gomborone JE, Dewsnap PA, Libby GW et al. Selective affective biasing in recognition memory in the irritable bowel syndrome. Gut 1993;34:1230-3.

48 Whitehead WE, Palsson OS. Is rectal pain sensitivity a biological marker for irritable bowel syndrome: psychological influences on pain perception. Gastroenterology 1998;115:1263-71.

49 Wade PR, Chen J, Jaffe B, et al. Localization and function of a 5-HT transporter in crypt epithelia of the gastrointestinal tract. $J$ Neurosci $1996 ; 16: 2352-64$

50 Nishizawa S, Benkelfat C, Young SN, et al. Differences between males and females in rates of serotonin synthesis in human brain. Proc Natl Acad Sci US A 1997;94:5308-13.

51 Jacobs BL, Fornal CA. Activity of serotonergic neurons in behaving animals. Neuropsychopharmacology 1999;21:9-15S

52 Lambert GW, Kaye DM, Cox HS, et al. Regional 5-hydroxyindoleacetic acid production in humans. Life Sci 1995;57:255-67.

53 Walther DJ, Bader M. A unique central tryptophan hydroxylase isoform. Biochem Pharmacol 2003:66:1673-80.

54 Walther DJ, Peter JU, Bashammakh S, et al. Synthesis of serotonin by a second tryptophan hydroxylase isoform. Science 2003;299:76. 